\title{
Functional Outcome of Primary Total Hip Arthroplasty using Harris Hip Score in Arthritic Hip
}

\author{
Suman Babu Marahatta ${ }^{1}$, Dirgha Raj $\mathrm{RC}^{2}$, Kapil Mani KC루 ${ }^{1}$ Arun Sigdel ${ }^{1}$
}

Author Info:

1Orthopaedic Surgeon, Civil Service Hospital and Assistant Professor, National Academy of medical Sciences, Kathmandu Nepal

${ }^{2}$ Professor National Academy Of Medical Sciences.

\section{Corresponding Author:}

Dr Suman Babu Marahatta, Registrar, Civil Service Hospital

Contact/Email: 9841154142 suman marahatta@hotmail.com https://orcid.org/0000-00029185-265X

\section{ABSTRACT}

Background: Total hip arthroplasty (THA) is a well-established procedure for advanced arthritis of hip joint. It significantly improves the quality of life by relieving pain and improving functional disability. The objective of this study was to evaluate clinical and functional outcome of primary total hip arthroplasty using Harris Hip Score.

Method: Prospective study was conducted in Civil Service Hospital. Out of 145 total hip arthroplasties performed from Jan 2014 to Dec 2018, first 100 cases that fulfilled the inclusion criteria were analyzed. Patient demographic including age, sex, site, operative indication and pre-operative Harris Hip Score were documented. Operative time, total intraoperative blood loss and complications were noted. Patients were followed in 3 weeks, 6 weeks, 3 months, 6 months and yearly. In each visit clinical evaluation using Harris Hip Score and radiological evaluation was done and documented. Duration of follow up ranged from minimum of 12 months to maximum 4.5 years.

Results: Age varied from 21 to 75 years, $59 \%$ were male and $41 \%$ female, right side involvement was seen in $55 \%$ and left side in $45 \%$. Major indication for surgery was avascular necrosis $46 \%$ and primary osteoarthritis in $24 \%$. Average operative time was 65 minutes and average intra operative blood loss was $655 \mathrm{ml}$. Pre-operative Harris Hip Score ranged from 25 to 59 with mean of 45.5 . The mean Harris hip score in last follow up increased to 90.5 with a minimum of 76 and maximum of 97 . Our study found that $85 \%$ had excellent, $9 \%$ had good and $6 \%$ had fair results. Complications include $2 \%$ dislocation, $1 \%$ infection, $1 \%$ greater trochanter avulsion and $1 \%$ screw irritation.

Conclusion: Primary Total hip arthroplasty is a safe and effective procedure. It improves pain and function hence improving activity of daily living and has fewer complications.

Keyword: Harris Hip Score; Outcome; Total Hip Arthroplasty.

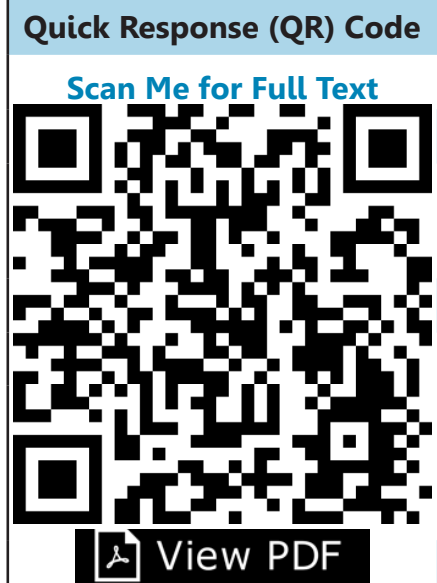

\section{Article Info}

Received: 24 May 2020; Accepted: 29 July 2020; Published Online: 30 July 2020 How to cite this article in Vancouver Style?

Marahatta SB, RC DR, KC KM, Sigdel A. Functional Outcome Of primary Total Hip Arthroplasty using Harris Hip Score in Arthritic Hip. Europasian J Med Sci.2020;2(2):4-10. https://doi.org/10.46405/ejms. $\underline{\mathrm{v} 2 \mathrm{i} 2.78}$

Conflict of Interest: None Declared;

Disclaimer

Source of Support: Nil

Copyright: (c) 2020 by author(s). This is an open access article distributed under the terms of the Creative Commons Attribution International License 4.0 (http://creativecommons.org/licenses/ by/4.0/) which permits unrestricted use, distribution, and reproduction in any medium, provided the original work is properly cited.

(2) View PDF

\section{Publisher's Note}

The Europasian Journal of Medical Sciences (EJMS) (www.europasianjournals.org) is an official Journal of Nirvana Psychosocial Care Center \& Ressearch Institute (www.nirvanapscc.com). The Journal as well as publisher remain neutral with regards to any jurisdictional claims in any published articles, its contents and the institutional affiliations of the authors. 


\section{INTRODUCTION}

Total Hip Arthroplasty (THA) is the most successful reconstructive procedure in orthopaedics. ${ }^{1}$ The success of THA is its ability to relieve the pain caused by end stage hip arthritis while maintaining the mobility and stability of the hip joint. Quality of life after THA is similar to that of a healthy reference group of similar age and sex. ${ }^{2}$ Those patients who are treated with surgery perform physically better than untreated patients with advanced hip osteoarthritis and the level of post-surgical satisfaction is very high. ${ }^{3}$ The incidence of hip arthritis is in increasing trend recently. Various causes that can lead to destruction of hip include primary osteoarthritis and secondary osteoarthritis due to osteonecrosis, inflammatory conditions like Rheumatoid arthritis, congenital hip causes like developmental dysplasia, post traumatic and post infectious conditions. End stage arthritis of the hip joint leads to incapacitating pain which hampers activity of daily living. THA is indicated when hip pain is not relieved by conservative measures like Non-steroidal anti-inflammatory drugs (NSAIDS), weight reduction, activity restriction, use of cane and lifestyle modification. Sir John Charnley in the 1960s designed artificial hip joints and laid down the principles of biomechanical hip joint. ${ }^{4}$ There have been several modifications in the design of implants since then but principles and basic concepts laid by him remain the same.

Conventional cemented total hip arthroplasty dramatically improves a patient's function and quality of life. Furthermore, with advanced prostheses design and modern cementing techniques, the rate of femoral loosening appears to be substantially reduced. ${ }^{5}$ Recently non cemented THA have taken over in recent times and have replaced conventional THA in young adults. Published results of THA have demonstrated excellent clinical, functional and radiographic outcomes in patients. ${ }^{6}$ These results, however, vary depending upon the type of implant, surgical technique, type of fixation, biomaterials, patient's age, cause of hip arthritis ${ }^{7}$ and numerous other factors. Various scoring systems have been developed to assess functional outcome of THA, among them Harris Hip Score is easy, reproducible and most commonly used scoring system. Harris Hip Score is calculated on the basis of pain domain based on pain severity and effect on activities and need for pain medications, functional domain based on daily activities and gait, deformity domain based on flexion, extension rotation and extremity length and motion domain based on range of motion of hip joint. ${ }^{8}$ Harris Hip Score is not only useful to predict outcome in total hip arthroplasty but the post-operative score change is also predictive of revision rates in post primary total hip arthroplasty cases. ${ }^{9}$ Studies suggest that THA is also one of the costeffective procedures in modern orthopaedics. ${ }^{10}$ Various complications have been documented during and after this procedure. Early complications include fracture in proximal femur and acetabulum, neurovascular injuries, dislocations, deep vein thrombosis and pulmonary embolism. Late complications include infection, loosening and heterotrophic ossification. Aseptic loosening of femoral or acetabular component which is a potential cause of pain and loss of function has evolved as the most serious complications of total hip arthroplasty and it accounts one of the most common cause of revision. ${ }^{11}$ Studies have suggested up to $90 \%$ success and good patient satisfaction in well performed Total hip Arthroplasty. ${ }^{12}$ Though Total Hip Arthroplasty has become a regular orthopaedic procedure in many tertiary centers of Nepal no such studies are done in our context. Our study aims to find functional outcome of primary THA using Harris Hip Score and assess complications related to this procedure.

\section{METHODOLOGY}

Prospective study was conducted in Civil Service Hospital from January 2014 to December 2018. Patient of any age group and sex with end stage arthritis of hip due to osteonecrosis, primary osteoarthritis, protrusio acetabuli grade 1 and DDH Crowe type 1 were included in the study. However, revision total hip arthroplasty, protrusio Grade 2 and 3, DDH Crowe 2, 3 and 4 and THA for fracture neck of femur and post girdlestone arthroplasty were excluded from the study. Out of 145 total hip arthroplasties performed, the first 100 cases that fulfilled the inclusion criteria were included in study and findings were analyzed using SPSS-16.

Patient demographic including age, sex, site, operative indication and pre-operative Harris Hip Score were documented. Operative time, total intraoperative blood loss and complications were noted. Patients were followed in 3 weeks, 6 weeks, 3 months, 6 months and yearly. In each visit clinical evaluation using Harris Hip Score and radiological evaluation was done and documented. Duration of follow up ranged from minimum of 12 months to maximum 4.5 years. Clinical results were evaluated using Harris Hip Score according to which out of total 100 points the score is reported as: 90-100 excellent results, 80-89 being good, 70-79 fair, 60-69 poor and below 60 a failed result.

Patients with advanced hip arthritis with severe pain hampering activity of daily living and not responding to conservative treatment were admitted from the outpatient department. Surgical procedure, outcome and possible complications that can occur during the surgery 
were well explained to the patients. Informed consent was taken before enrolling the patient in the study. Ethical approval for the study was taken from IRC, Civil Service Hospital.

X-ray both hips anteroposterior and lateral views, $\mathrm{X}$-ray of spine anteroposterior and lateral views were performed. Patient demographic data including age, sex, site, operative indication and pre-operative Harris Hip Score calculated. All patients were operated in lateral position with modified Hardinge lateral approach with anterior dislocation of hip. Preoperative antibiotic ceftriaxone $1 \mathrm{gm}$ given intravenous half an hour prior to skin incision. All patients received regional anesthesia with lumbosacral plexus block. Acetabular and femoral preparation was done in standard fashion and trial implants were inserted. Stability of the hip joint was assessed and final implant placed. We were able to use an uncemented acetabulum with 2 screws and uncemented femoral component in all cases. Meticulous closure of capsule, gluteus medius and tensor fascia lata was done with suction drain which was inflated 2 hours after surgery. Patients were mobilized from the second postoperative day on crutches and put on toe touch bearing for 6 weeks. Patients received 5 days of intravenous antibiotics followed by 2 weeks of oral antibiotics at the time of discharge. Patients were put on standard physiotherapy protocol. They were followed in standard time and in each visit clinical evaluation and Harris Hip Score was calculated and documented.

\section{RESULTS}

Age varied from minimum of 21 years and maximum of 75 years with mean age of 48 years (SD 9.56). Majority of the patients were male $(59 \%)$ and $(41 \%)$ were female. Right side involvement was more common (55\%) as

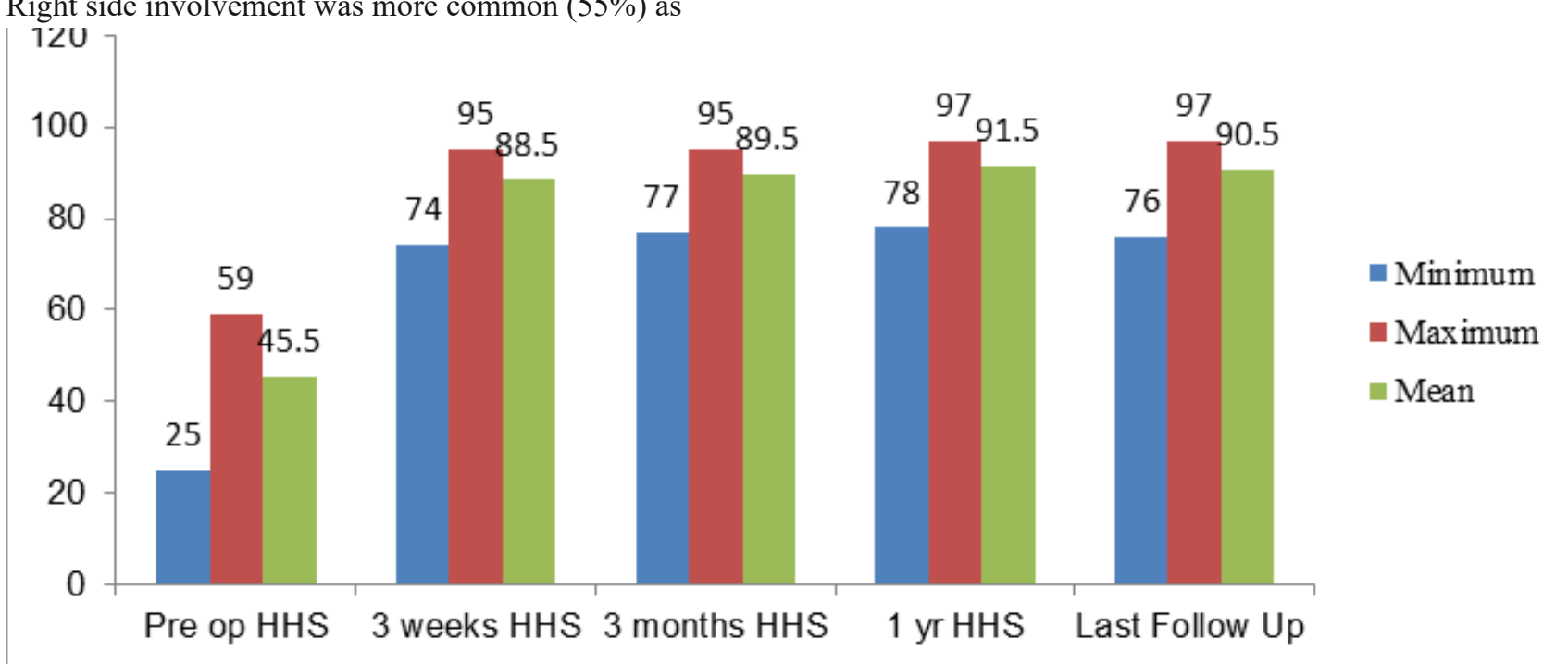

Figure 3: Comparison of Preoperative and postoperative Harris Hip Score in patients undergoing Total Hip Arthroplasty

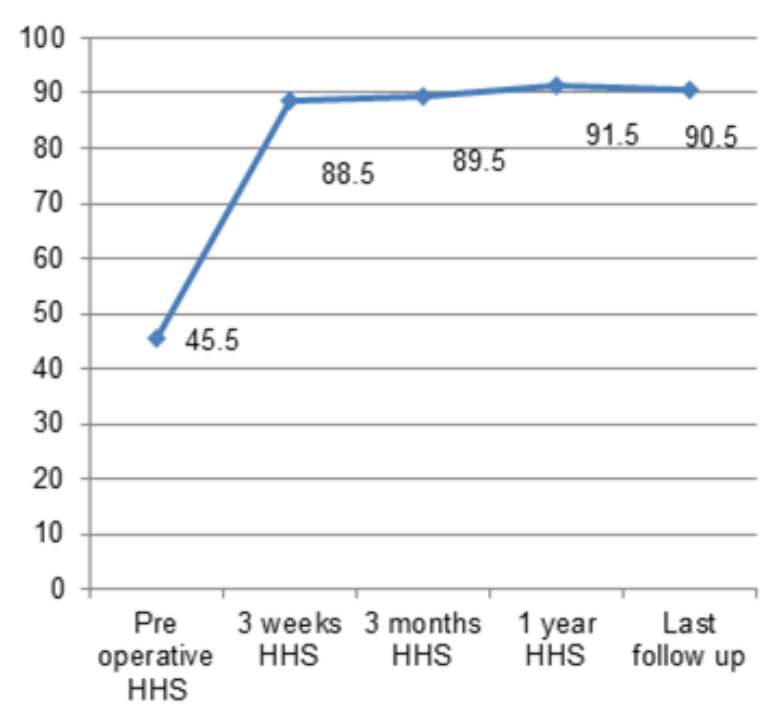

Figure 1: Graphical representation of improvement of Harris Hip Score of patient

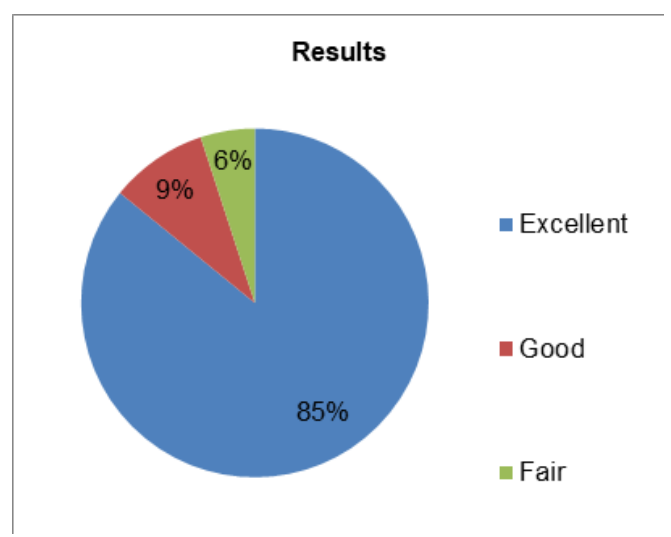

Figure 2: Functional outcome of patients undergoing Total Hip Arthroplasty $(\mathrm{N}=100)$ 
compared to left side (45\%). Major indication for surgery was avascular necrosis $(36 \%)$ followed by primary osteoarthritis $(24 \%)$, post infection $(12 \%)$ and other indications like post trauma, rheumatoid arthritis and developmental dysplasia of hip (28\%).

Average operative time was 65 minutes with minimum of 55 minutes and maximum of 85 minutes. Intra operative blood loss varied from $350 \mathrm{ml}$ to $800 \mathrm{ml}$ with an average of $650 \mathrm{ml}$. Pre-operative Harris Hip Score ranged from 25 to 59 with mean of 45.5. 3 weeks postoperative Harris hip score was 88.44 with a minimum of 74 and maximum of 95 . The mean followup Harris hip score in 3 months and 1year increased to 89.5 and 91.5 with a minimum of 77 and maximum of 97 . The mean score in last follow up was 90.5 with minimum of 76 to maximum of 97 (Figure 2 and 3). Our study showed $85 \%$ excellent, $9 \%$ good and $6 \%$ having fair results postoperatively which is graded according to Harris Hip Score (Figure 2).

Regarding the complications $2 \%$ of cases developed superficial skin infection. Two cases $(2 \%)$ developed dislocation in an early post-operative period that was managed with closed reduction and traction for 6 weeks. One patient (1\%) developed screw irritation at sciatic nerve. The screw was removed after taking out the liner. Patient got better after revision surgery with no residual effects. One patient (1\%) developed greater trochanter avulsion intraoperatively managed by circlage with SS wire and delayed weight bearing.

\section{Clinical Pictures Related to our Study}

A 28 years male with primary AVN of right hip sterioid induced for renal problesm Pre-operative HHS 45, rative HHS 45, increased to 90 after 3 months.

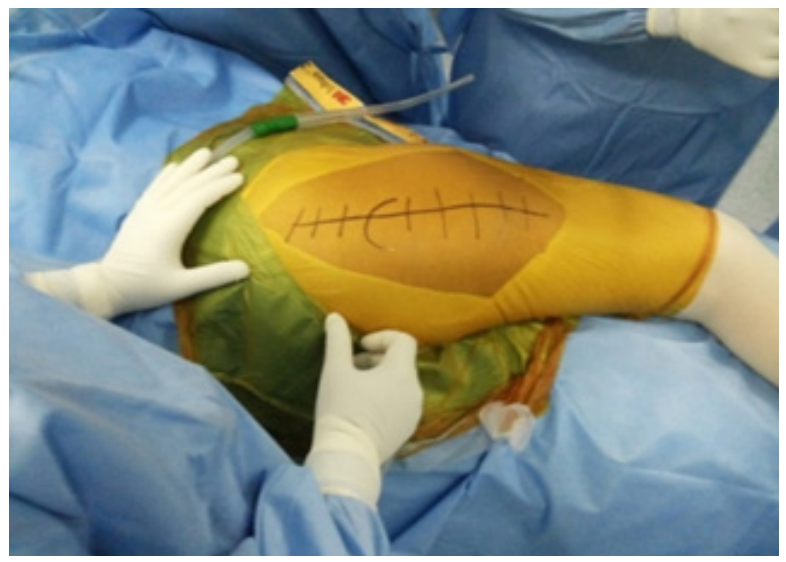

Photograph 1: Patient Positioning

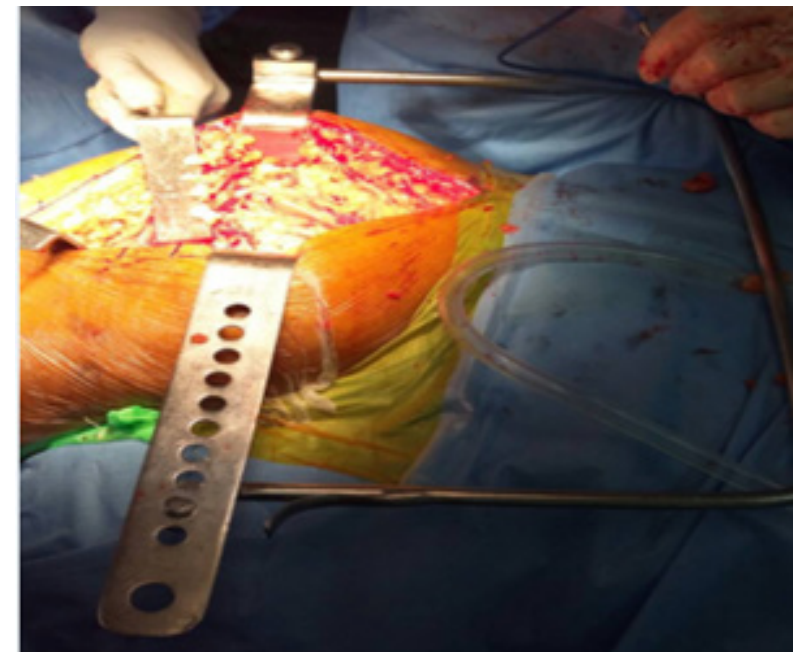

Photograph 2: Use of Charnley Retractor

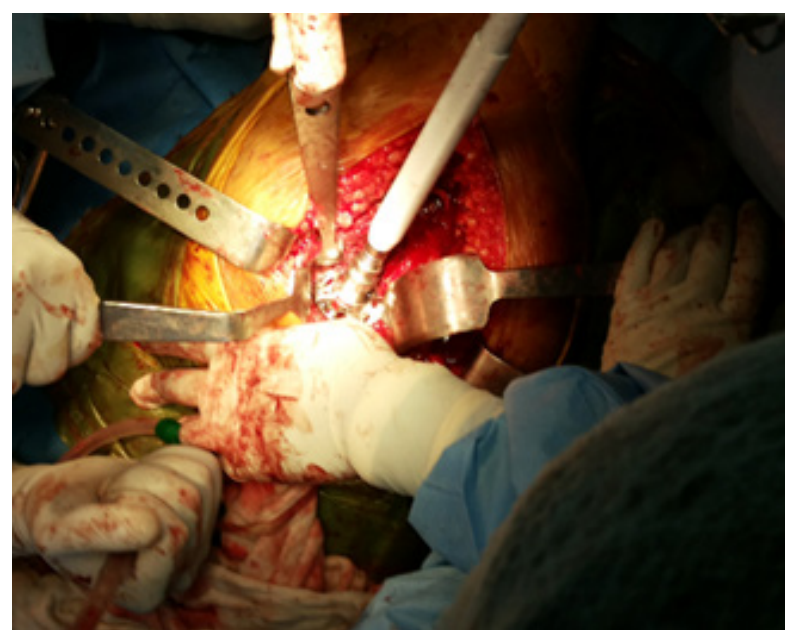

Photograph 3: Acetabular Preperation

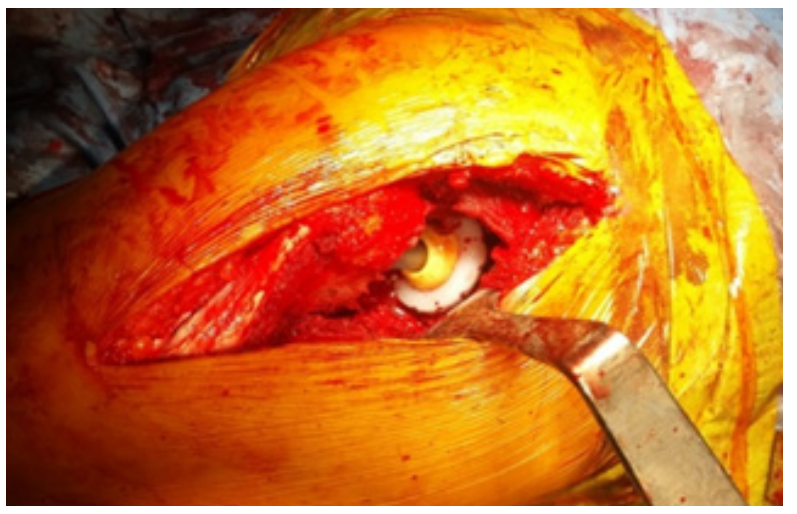

Photograph 4: Ranawat coplanar test 


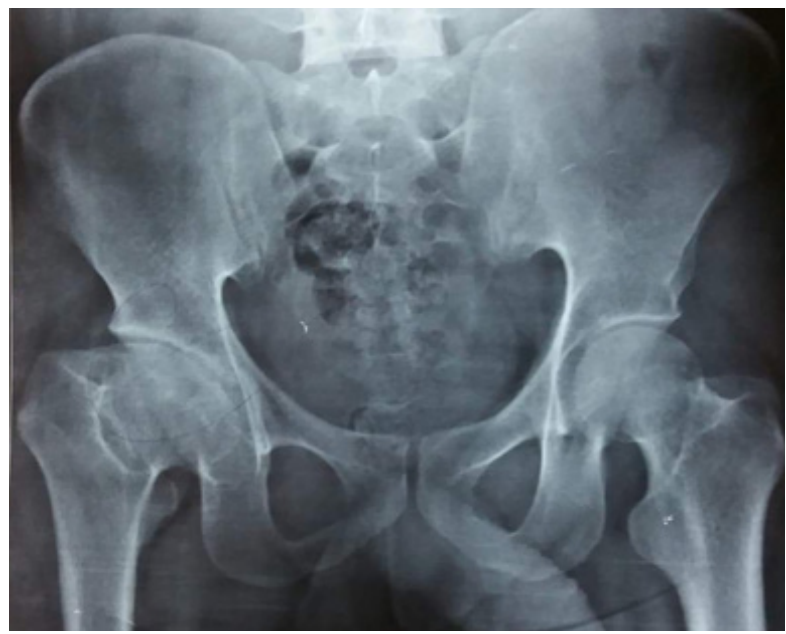

Photograph 5a: Preoperative AP X-Ray

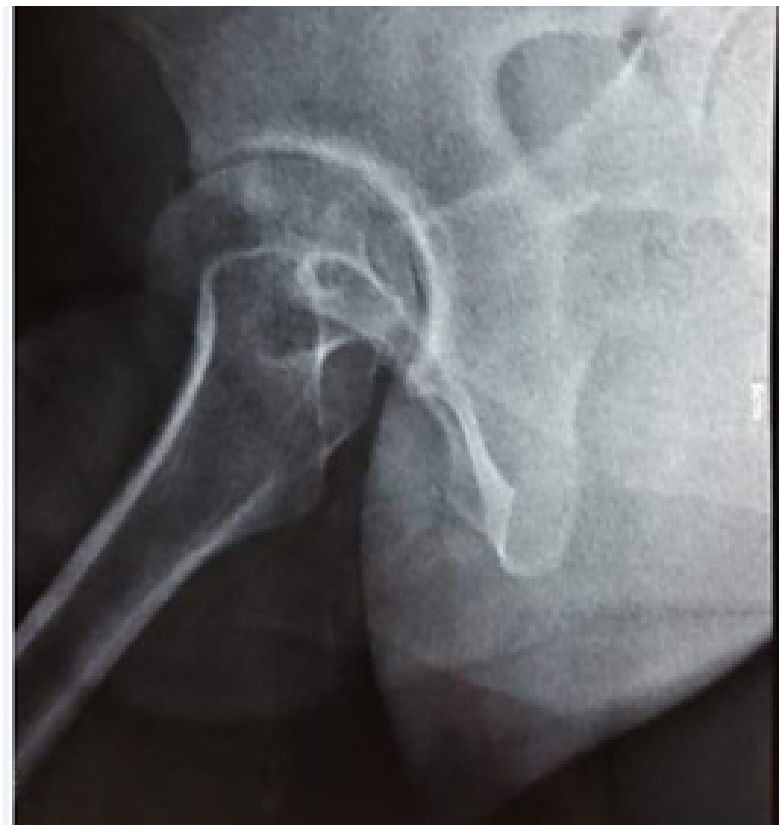

Photograph 5b: Pre-operative Lateral X-Ray

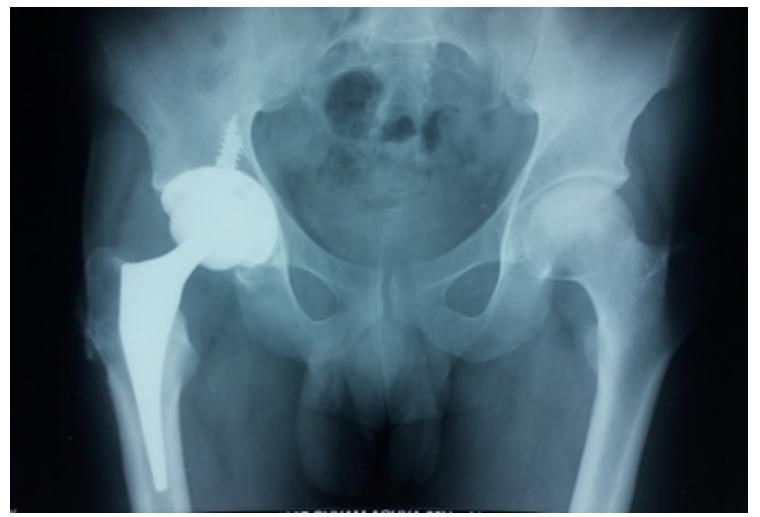

Photograpg 6: Nine-months post OP X- Ray
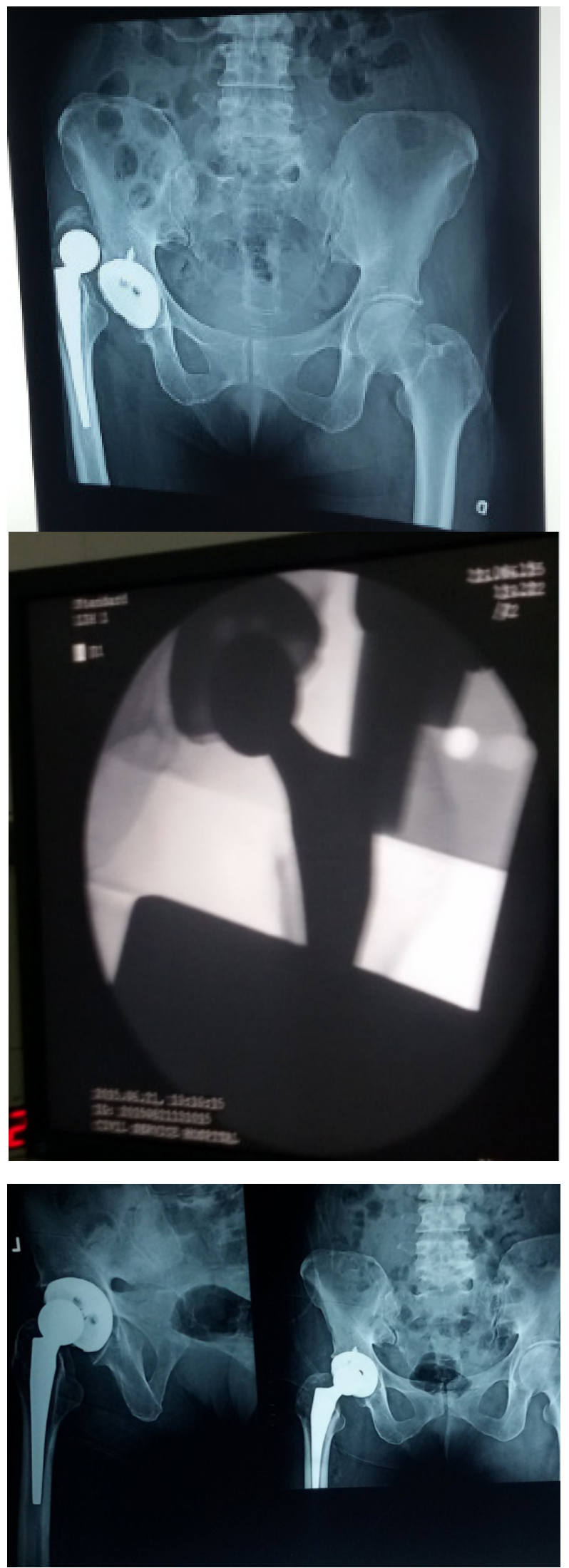

Photograph 7: Pre and post reduction X-Ray of hip dislocation of THA patient 


\section{DISCUSSION}

Total hip Arthroplasty is a well-established and most common adult reconstructive procedure in orthopaedics. It relieves pain and restores normal function of the hip that is destroyed by various conditions. Patients after Total Hip Arthroplasty regain normal or near normal life with good patient satisfaction. The evolution of arthroplasty in recent years has focused on prolonging the durability of implants, especially as the population of patients expected to require THA expands. Perioperative pain management protocols and accelerated rehabilitation have improved the ability of patients to recover after the operation ${ }^{2}$.

The age of the patient varied from minimum of 21 years to maximum of 75 years with mean age of 48 years. In a similar study ${ }^{11}$ where 100 cases were included in the study the average age of the patient was 52.56. Similarly, in another study conducted by A. Chandra Sekhar et al $^{12}$ the average age of patients was 59.68 years. The studies showed variation in the age group which clearly indicates that age is not the indication for THA. Patients of any age with end stage arthritis are candidates for THA.

In our study we found that the percentage of male patients $(59 \%)$ was slightly higher than female (41\%). Several other studies also found that THA is commonly ${ }^{12,13}$ performed in males as compared to females. Major indication for surgery in our study was osteonecrosis (36\%) followed by primary osteoarthritis $24 \%$, post infectious $12 \%$ and $28 \%$ others (post trauma, RA, DDH). Similar study ${ }^{12}$ conducted in our region including India also highlighted that osteonecrosis is a common cause of hip arthritis needing total hip arthroplasty. However, studies from other parts ${ }^{14}$ have found that primary osteoarthritis is a common indication for total hip arthroplasty. The reason for primary osteoarthritis of the hip is less common in our part as compared to western and other parts of the world could be due to our cultural habits and day to day activities. We have a habit of sitting cross legs and squatting as compared to western population so primary osteoarthritis is common in knees as compared to hips. Similarly sickle cell disease is common in certain communities in our country and the institute where this study was conducted is a tertiary referral centre for hematological diseases. This may be the reason for getting more cases of avascular necrosis as compared to primary osteoarthritis.

We used Harris Hip Score to calculate functional outcomes of our patients. Pre-operative Harris Hip Score ranged from 25 to 59 with a mean of 45.5. Mean three weeks postoperative Harris hip score was 88.44 with a minimum of 74 and maximum of 95 . The mean follow-up Harris hip score in three months and one year increased to 89.5 and 91.5 with a minimum of 77 and maximum of 97 . The mean score in last follow up was 88.5 with minimum of 76 to maximum of 97 . Our findings were similar to several other studies. In a similar study conducted by Ganesan et $\mathrm{al}^{7}$ where they compared the functional outcome of total hip arthroplasty in osteoarthritis and rheumatoid arthritis in 44 hips of 44 patients. The mean preoperative and latest Harris hip score were 44 and 88 respectively. The mean Harris hip score at the end of $1 \mathrm{st}$, 3rd and 5th years follow up were 86,87 and 87 respectively. Our results were quite similar to the study conducted by Gupta et al ${ }^{15}$ where average modified HHS was 28 preoperatively, increased to 64 at 6 weeks, 86 at six months, 90 at one year and 92 at the last follow-up. After one year of follow up, modified HHS was excellent in 17 patients and good in 3 patients. Similarly, in another study conducted by Siddique et $\mathrm{al}^{13}$ where they conducted study in 65 primary hips HHS was calculated for all patients at $2^{\text {nd }}$ week, $1^{\text {st }}$ month and $6^{\text {th }}$ month post-operatively. Preoperative mean HHS was $41.15 \pm 5.12$ (range: $29-49$ ). At 2 nd week, the mean HHS was $58.76 \pm 4.57$ (range: $46-69)$ ). At 1 month mean HHS was $68.13 \pm 4.55$ (range: $52-78$ ). At 6month, mean HHS was $78.64 \pm 2.28$ (range: 73-86). These results are quite similar with our findings. Studies also showed that there was a persistent increase in Harris Hip Score from immediate postoperative period to initial 6 months where the scores were highest and there was a plateau after that time period. In our study there was a significant improvement in post-operative Harris hip score with $85 \%$ excellent, $9 \%$ good and $6 \%$ having fair results. Our results were consistent with several other studies. ${ }^{7,14,15}$

Various complications were encountered in our study. $2 \%$ of cases developed superficial skin infection that got better with antibiotics for 6 weeks. Two cases $(2 \%)$ developed dislocation in the early post-operative period within 3 weeks of operation. Both patients had a history of trivial fall that led to anterior dislocation of hip in both cases. Patients were managed with closed reduction and traction for 6 weeks. Patients were advised normal activities after which there were no residual difficulties. Our dislocation rate was similar to several other studies. ${ }^{2,13,14}$ Most of the studies however report similar dislocation rates but they have posterior dislocation the reason being the posterior approach is used in most of the studies. We encountered very unusual complications in one patient where a patient developed screw irritation on the sciatic nerve. This was a case of rheumatoid arthritis where screws were used for the additional stability for the acetabular cup. Patient developed severe pain and tingling sensation along the sciatic nerve area after the block was over. Screw was removed and placed on another place after taking out 
the liner. Patient got better after revision surgery with no residual effects. This is a very unusual complication and we recommend to follow the rules of safe quadrant while placing screws as well as we encourage use of image intensifier if in case the surgeon is not sure about the placement of screws. One patient $(1 \%)$ developed greater trochanter avulsion intraoperatively. The greater trochanter was undisplaced and the implant was stable on our intraoperative evaluation. This was managed by circlage with SS wire and weight bearing was delayed for 6 weeks. Several studies ${ }^{11,15}$ have found osteolysis, aseptic loosening and symptomatic failure of both acetabular and femoral components in their study. We did not encounter such complications. The reason behind this could be these are late complications which are usually encountered after 5 years and our study time is shorter. There was no mortality noted in our study.

\section{CONCLUSION}

Total Hip Arthroplasty is a safe and effective procedure that relieves pain and improves quality of daily living in a patient with end stage arthritis of the hip. It has got excellent functional outcome and less complication.

\section{REFERENCES}

1. Campbell's Operative Orthopaedics - 12th Edition [Internet]. [cited 2020 Apr 15]. Available from: https:// www.elsevier.com/books/campbells-operativeorthopaedics/canale/978-0-323-07243-4.[Pubmed] [Google Scholar]

2. Wiklund I, Romanus B. A comparison of quality of life before and after arthroplasty in patients who had arthrosis of the hip joint. J Bone Joint Surg Am. 1991 Jun;73(5):765-9. [Pubmed] [Google Scholar]

3. Mariconda M, Galasso O, Costa GG, Recano P, Cerbasi S. Quality of life and functionality after total hip arthroplasty: a long-term follow-up study. BMC Musculoskelet Disord. 2011 Oct 6;12(1):222. [PubMed] [Google Scholar]

4. Charnley J. ARTHROPLASTY OF THE HIP: A New Operation. The Lancet. 1961 May 27;277(7187):112932. [Pubmed] [Google Scholar]

5. Park KD, Park JB. Interfacial strength of compressionmolded specimens between PMMA powder and PMMA/ MMA monomer solution-treated ultra-high molecular weight polyethylene (UHMWPE) powder. J Biomed Mater Res. 2000;53(6):737-47. [Google Scholar]

6. Callaghan JJ, Dysart SH, Savory CG. The uncemented porous-coated anatomic total hip prosthesis. Two-year results of a prospective consecutive series. J Bone Joint Surg Am. 1988 Mar;70(3):337-46. [Pubmed] [Google Scholar]

7. Ram GG, Thamodaran B, Ashok TR, Perumal S, Varthi VP. Analysis of Functional and Radiological Outcome of Total Hip Replacements in Rheumatoid and
Osteoarthritis Patients*. Open Journal of Rheumatology and Autoimmune Diseases. 2013 Nov 19;3(4):720-6. http:/dx.doi.org/10.4236/ojra.2013.34038

8. Harris Hip Score [Internet]. Physiopedia. [cited 2020 Apr 16]. Available from: https://www.physio-pedia.com/ Harris_Hip_Score

9. Singh JA, Schleck C, Harmsen S, Lewallen D. Clinically important improvement thresholds for Harris Hip Score and its ability to predict revision risk after primary total hip arthroplasty. BMC Musculoskeletal Disorders. 2016 Jun 10;17(1):256. [Pubmed] [Google Scholar]

10. Faulkner A, Kennedy LG, Baxter K, Donovan J, Wilkinson M, Bevan G. Effectiveness of hip prostheses in primary total hip replacement: a critical review of evidence and an economic model. Health Technol Assess. 1998;2(6):1-133. [Pubmed] [Google Scholar]

11. Siwach R, Kadyan VS, Sangwan S, Gupta R. A retrospective study of total hip arthroplasty. Indian J Orthop. 2007;41(1):62-6. [PubMed] [Google Scholar]

12. Sekhar AC, Mittal A, Rallapalli R, Biju, Prasad S. Evaluation and Outcome of Total Hip Replacement in Adults with Arthritis. In 2015. IOSR Journal of Dental and Medical Sciences (IOSR-JDMS) e-ISSN: 22790853, p-ISSN: 2279-0861.Volume 14, Issue 4 Ver. VIII (Apr. 2015), PP 65-72

13. Siddique T, Sah RK, Masood F, Awais SM. Improvement in Harris Hip Score after cementless total hip arthroplasty in young active adults with secondary hip arthritis- A short-term follow-up result. J Pak Med Assoc. 2015 Nov;65(11 Suppl 3):S63-66. [Google Scholar]

14. Katchy AU, Katchy SC, Ekwedigwe H, Ezeobi I. Total hip replacement for management of severe osteoarthritis in a developing country: A 5-year assessment of functional outcome in 72 consecutive hip. Nigerian Journal of Orthopaedics and Trauma. 2018 Jul 1;17(2):46. [Google Scholar]

15. Gupta DL, Lal DM, Aggarwal DV, Rathor DLP. Assessing functional outcome using modified Harris hip score in patients undergoing total hip replacement. International Journal of Orthopaedics Sciences. 2018 Apr $1 ; 4(2): 1015-7$. 